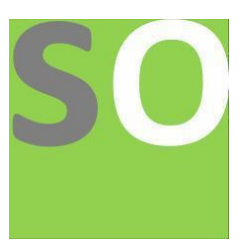

Article title: Integrated Design of Adaptive \& Fuzzy Logic Control for Trajectory Tracking of 2 DOF Quadrotor Authors: Mustefa Jibril[1]

Affiliations: Dire Dawa University, Dire Dawa, Ethiopia[1]

Orcid ids: 0000-0002-3165-2410[1]

Contact e-mail: mustefazinet1981@gmail.com

License information: This work has been published open access under Creative Commons Attribution License $\mathrm{http}: / / c r e a t i v e c o m m o n s . o r g / l i c e n s e s / b y / 4.0 /$, which permits unrestricted use, distribution, and reproduction in any medium, provided the original work is properly cited. Conditions, terms of use and publishing policy can be found at https://www.scienceopen.com/.

Preprint statement: This article is a preprint and has not been peer-reviewed, under consideration and submitted to ScienceOpen Preprints for open peer review.

DOI: 10.14293/S2199-1006.1.SOR-.PPOXJNG.v1

Preprint first posted online: 27 September 2021

Keywords: Quadrotor, Adaptive fuzzy, Fuzzy logic, PID 


\title{
Integrated Design of Adaptive \& Fuzzy Logic Control for Trajectory Tracking of 2 DOF Quadrotor
}

\author{
Mustefa Jibril \\ School of Electrical \& Computer Engineering, Dire Dawa Institute of Technology, Dire Dawa, \\ Ethiopia \\ mustefa.jibril@ddu.edu.et
}

\begin{abstract}
Accurate and precise trajectory tracking is crucial for a quadrotor to operate in disturbed environments. This paper presents a novel tracking hybrid controller for a quadrotor UAV that combines the Adaptive and Fuzzy logic controller. The Adaptive fuzzy controller is implemented to govern the behavior of two degrees of freedom quadrotor UAV. The proposed controller allows controlling the movement of UAVs to track a given trajectory in a $2 \mathrm{D}$ vertical plane. The Fuzzy Logic system provides an automatic adjustment of the Adaptive parameters to reduce tracking errors and improve the quality of the controller. The results showed perfect behavior for the control law to control a quadrotor trajectory tracking task. To show the effectiveness of the intelligent controller, simulation results are given to confirm the advantages of the proposed control method, compared with Fuzzy and Proportional integral derivative (PID) control methods.
\end{abstract}

Keywords: Quadrotor, Adaptive fuzzy, Fuzzy logic, PID

\section{Introduction}

The quadrotor or quadcopter is a unique kind of unmanned aerial vehicle (UAV) that has Vertical Take-Off and Landing ability [1]. The quadrotor has a bonus of maneuverability because of its inherently dynamic nature [2]. Quadrotors commonly have rotors spinning clockwise (CW) and counterclockwise $(\mathrm{CCW})$. Flight management is furnished through an unbiased variant of the velocity and for this reason raise and torque of every rotor [6]. Pitch and roll are managed through various internet middle of thrust, with yaw managed through various internet torque.

Unlike traditional helicopters, quadrotors do now no longer typically have cyclic pitch management, wherein the attitude of the blades varies dynamically as they flip around the rotor hub [4]. In the early days of flight, quadrotors (then mentioned both as quadrotors or really as helicopters) have been visible as a likely answer to several chronic issues in vertical flight. Torquebrought about manage problems (in addition to performance problems originating from the tail rotor, which generates no beneficial raise) may be removed through counter-rotation, and the incredibly brief blades are plenty simpler to construct. A wide variety of manned designs is regarded withinside the Twenties and 1930s. These motors have been the various first-a-hit heavier-than-air vertical take-off and landing (VTOL) motors. However, early prototypes suffered from negative performance, and latter prototypes required an excessive amount of pilot paintings load, because of negative balance augmentation and constrained management authority.

In the closing few decades, the quadrotor format has to turn out to be famous for small-scale unmanned aerial automobiles or drones. The want for a plane with more maneuverability and soaring capacity has brought about an upward thrust in quadrotor research [5]. The four-rotor layout lets in quadrotor to be exceedingly easy in layout but notably reliable and maneuverable. 
Research is persevering to boom the talents of quadrotors through making advances in multi-craft communication, surroundings exploration, and maneuverability. If those growing characteristics may be combined, quadrotors might be able to superior self-sufficient missions which might be present now no longer viable with different automobiles [6].

For small drones, quadrotors are less expensive and greater long-lasting than traditional helicopters due to their mechanical simplicity. Their smaller blades also are advantageous due to the fact they own much less kinetic energy, decreasing their capacity to cause damage [9]. For small-scale quadrotors, this makes the automobiles more secure for close interaction. It is likewise viable to in-shape quadrotors with guards that enclose the rotors, similarly decreasing the ability for damage. However, as length will increases, constant propeller quadrotors broaden risks relative to traditional helicopters. Increasing blade length will increase their momentum [7]. This means that adjustments in blade pace take longer, which negatively affects control. Helicopters do now no longer enjoy this trouble as growing the dimensions of the rotor disk does now no longer notably affect the capacity to govern blade pitch.

\section{Dynamic Modelling of Quadrotor}

A quadrotor version has been designed with 2 propellers which are pushed with the aid of using DC motors. The front propeller controls the elevation of the quadrotor nostril approximately the pitch axis and the again propeller controls the aspect-to-aspect motions of the quadrotor approximately the yaw axis [10]. The free-body diagram of the 2 DOF quadrotor is shown in Figure 1 below.

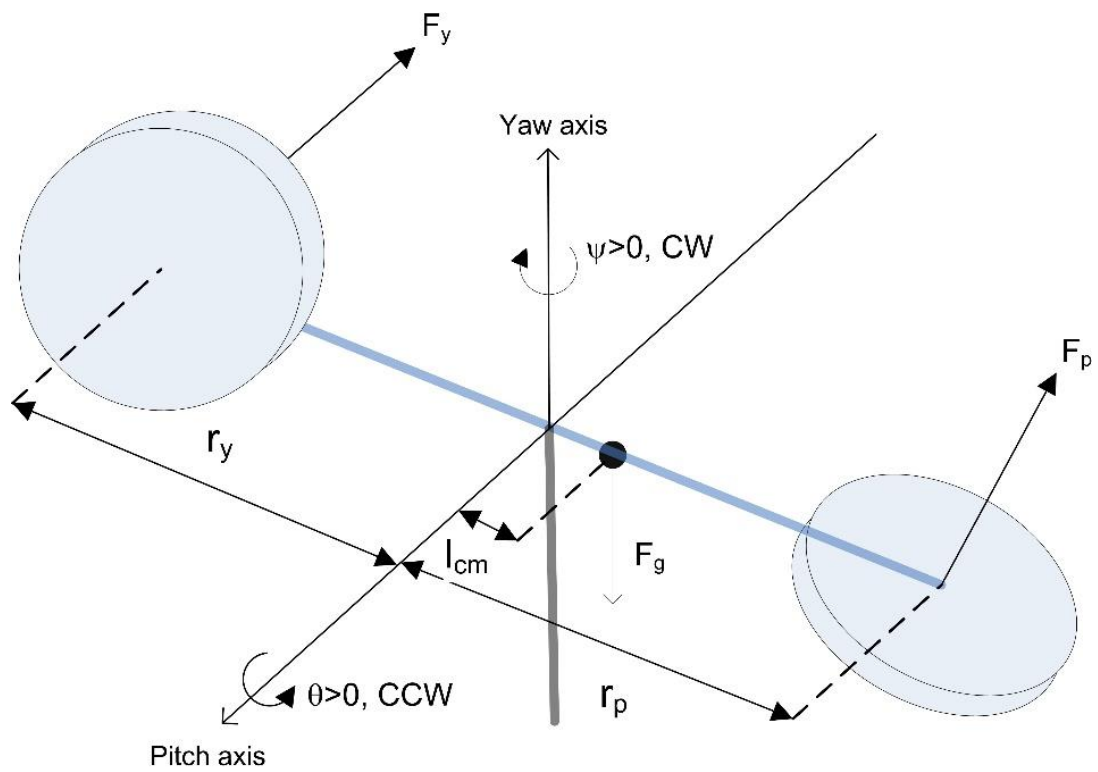

Figure 12 DOF quadrotor

\subsection{Generalized Coordinates: $q[i]$ 's}

In analytical mechanics, the term generalized coordinates refer back to the parameters that describe the configuration of the machine relative to a few reference configurations. These parameters ought to uniquely outline the configuration of the machine relative to the reference configuration. 
The generalized coordinates are also called Lagrangian coordinates. In this model the two coordinates are:

$\alpha(t)=$ pitch angle

$\beta(t)=$ yaw angle

Which expressed as:

$$
q=[\alpha(t), \beta(t)]
$$

The derivative of the coordinates become

$$
q_{d}=\left[\frac{d \alpha(t)}{d t}, \frac{d \beta(t)}{d t}\right]
$$

Let

$$
\alpha(t)=X_{1}, \beta(t)=X_{2}, \dot{\alpha}(t)=X_{3}, \dot{\beta}(t)=X_{4}, \ddot{\alpha}(t)=\dot{X}_{3}, \ddot{\beta}(t)=\dot{X}_{4}, u_{1}=V_{m_{-} p}, u_{2}=V_{m_{-} y}
$$

\subsection{Total Potential and Kinetic Energies of the System}

Energy stored in an item because of its position is Potential Energy. The energy that a transferring item has because of its movement is Kinetic Energy. The total potential and kinetic energies are needed to calculate the Lagrangian of the system.

\subsubsection{Total Potential Energy: $V_{T}$}

The total potential energy may be expressed in phrases of the generalized coordinates as.

$V_{g}=$ potential energy of quadrotor.

$$
V_{g}=m_{\text {quad }} g \sin (\alpha(t)) l_{c m}
$$

And the total potential energy of the system

$$
V_{T}=m_{\text {quad }} g \sin (\alpha(t)) l_{c m}
$$

\subsubsection{Total Kinetic Energy: $T_{T}$}

The total kinetic energy may be expressed in phrases of the generalized coordinates and their firstderivatives as.

$T_{r l}=$ kinetic energy due to rotation of the arm

$J_{m}=$ moment of inertia of the motor and rotary arm. 


$$
\begin{aligned}
& T_{r 1}=\frac{1}{2} J_{e q_{-} \text {Pitch }}\left[\frac{d \alpha(t)}{d t}\right]^{2} \\
& T_{r 2}=\frac{1}{2} J_{\text {eq_yaw }}\left[\frac{d \beta(t)}{d t}\right]^{2} \\
& T_{J}=\frac{1}{2} m_{\text {quad }}\left[\begin{array}{l}
{\left[-\sin (\beta(t))\left[\frac{d \beta(t)}{d t}\right] \cos (\alpha(t)) l_{c m}-\cos (\beta(t)) \sin (\alpha(t))\left[\frac{d \alpha(t)}{d t}\right] l_{c m}\right]^{2}} \\
+\left[-\cos (\beta(t))\left[\frac{d \beta(t)}{d t}\right] \cos (\alpha(t)) l_{c m}+\sin (\beta(t)) \sin (\alpha(t))\left[\frac{d \alpha(t)}{d t}\right] l_{c m}\right]^{2}+\cos (\alpha(t))^{2} l_{c m}^{2}
\end{array}\right]
\end{aligned}
$$

Total kinetic energy

$$
\begin{aligned}
& T_{T}=\frac{1}{2} J_{\text {eq_P Pich }}\left[\frac{d \alpha(t)}{d t}\right]^{2}+\frac{1}{2} J_{\text {eq_yaw }}\left[\frac{d \beta(t)}{d t}\right]^{2}+ \\
& \frac{1}{2} m_{\text {quad }}\left[\begin{array}{l}
{\left[-\sin (\beta(t))\left[\frac{d \beta(t)}{d t}\right] \cos (\alpha(t)) l_{c m}-\cos (\beta(t)) \sin (\alpha(t))\left[\frac{d \alpha(t)}{d t}\right] l_{c m}\right]^{2}} \\
+\left[-\cos (\beta(t))\left[\frac{d \beta(t)}{d t}\right] \cos (\alpha(t)) l_{c m}+\sin (\beta(t)) \sin (\alpha(t))\left[\frac{d \alpha(t)}{d t}\right] l_{c m}\right]^{2}+\cos (\alpha(t))^{2} l_{c m}^{2}
\end{array}\right.
\end{aligned}
$$

\subsection{Generalized Forces: $Q_{i}$ 's}

Generalized forces locate used in Lagrangian mechanics, wherein they play a function conjugate to generalized coordinates. They are acquired from the carried-out forces, $\mathrm{Fi}, \mathrm{i}=1 \ldots, \mathrm{n}$, performing on a system that has its configuration described in phrases of generalized coordinates. In the components of digital work, every generalized pressure is the coefficient of the version of a generalized coordinate. The non-conservative forces corresponding to the generalized coordinates are: $\tau_{m}$ and the viscous damping forces $B_{m}$,

Where

$\tau_{p}=$ torque acting on pitch axis

$\tau_{y}=$ torque acting on yaw axis

$B_{p}=$ pitch viscous friction torque coefficient (a.k.a. viscous damping)

$B_{y}=$ yaw viscous friction torque coefficient (a.k.a. viscous damping)

$\mathrm{Q}[\mathrm{i}]=$ generalized force applied on generalized coordinate $\mathrm{q}[\mathrm{i}]$ 


$$
\begin{aligned}
& Q_{1}=\tau_{p}-B_{p}\left[\frac{d \alpha(t)}{d t}\right] \\
& Q_{2}=\tau_{y}-B_{y}\left[\frac{d \beta(t)}{d t}\right]
\end{aligned}
$$

For the input $u=\left[\tau_{p}, \tau_{y}\right]$

$$
\begin{aligned}
& \tau_{p}=K_{p p} V_{m_{-} p}+K_{p y} V_{m_{-} y} \\
& \tau_{y}=K_{y y} V_{m_{-} y}+K_{y p} V_{m_{-} p}
\end{aligned}
$$

Where

$K_{p p}$ Torque constant on pitch-pitch axis

$K_{p y}$ Torque constant on pitch-yaw axis

$K_{y y}$ Torque constant on yaw-yaw axis

$K_{y p}$ Torque constant on yaw-pitch axis

$V_{m_{-} y}$ yaw motor voltage

$V_{m_{-} p}$ pitch motor voltage

Where Q becomes

$$
Q=\left[K_{p p} V_{m_{-} p}+K_{p y} V_{m_{-} y}-B_{p}\left[\frac{d \alpha(t)}{d t}\right], K_{y y} V_{m_{-} y}+K_{y p} V_{m_{-} p}-B_{y}\left[\frac{d \beta(t)}{d t}\right]\right]
$$

\subsection{Euler-Lagrange's Equations}

In the calculus of variations and classical mechanics, the Euler-Lagrange equation is a gadget of second-order ordinary differential equations whose solutions are stationary points of the given action functional. For an $N$-DOF system, Lagrange's equations can be written:

$$
\left[\frac{\partial}{\partial t}\left[\frac{\partial}{\partial \dot{q}_{i}} L\right]\right]-\left[\frac{\partial}{\partial q_{i}} L\right]=Q_{i} \text { for } i=1 \text { to } i=N
$$


where:

$Q_{i}$ 's are special combinations of external forces and called the generalized forces $q_{1} \ldots . . . . . . q_{N}$, are $N$ independent coordinates chosen to describe the system and called the generalized coordinates, and $\mathrm{L}$ is the Lagrangian of the system. $\mathrm{L}$ is defined by:

$$
\mathrm{L}=\mathrm{T}-\mathrm{U}
$$

where $\mathrm{T}$ is the total kinetic energy of the system and $\mathrm{U}$ the total potential energy of the system.

\subsection{Inertia (or mass) Matrix: Fi}

In analytical mechanics, the force matrix is a symmetric matrix $M$ that expresses the relationship between the time derivative of the generalized coordinate vector $\mathrm{q}$ of a machine and the kinetic energy $\mathrm{T}$ of that system. The linear model of the inertia matrix for small-displacements

$$
F=\left(\begin{array}{cc}
J_{p}+m_{\text {quad }} l_{c m}{ }^{2} & 0 \\
0 & J_{y}+m_{\text {quad }} l_{c m}{ }^{2}
\end{array}\right)
$$

\subsection{State-space System Model}

The state-space representation of the system is

$$
A=\left(\begin{array}{cccc}
0 & 0 & 1 & 0 \\
0 & 0 & 0 & 1 \\
0 & 0 & -\frac{B_{p}}{J_{p}+m_{\text {quad }} l_{c m}^{2}} & 0 \\
0 & 0 & 0 & -\frac{B_{y}}{J_{y}+m_{\text {quad }} l_{c m}^{2}}
\end{array}\right) \quad B=\left(\begin{array}{cc}
0 & 0 \\
0 & 0 \\
\frac{K_{p p}}{J_{p}+m_{\text {qual }} l_{c m}^{2}} & \frac{K_{p y}}{J_{p}+m_{\text {quad }} l_{c m}^{2}} \\
\frac{K_{y p}}{J_{y}+m_{\text {quad }} l_{c m}^{2}} & \frac{K_{y y}}{J_{y}+m_{\text {quad }} l_{c m}^{2}}
\end{array}\right) \quad C=\left(\begin{array}{cccc}
1 & 0 & 0 & 0 \\
0 & 1 & 0 & 0 \\
0 & 0 & 1 & 0 \\
0 & 0 & 0 & 1
\end{array}\right) \quad D=\left(\begin{array}{cc}
0 & 0 \\
0 & 0 \\
0 & 0 \\
0 & 0
\end{array}\right)
$$

The parameters of the 2 DOF quadrotor are shown in Table 1 below.

Table 1 Quadrotor parameters

\begin{tabular}{|l|l|l|l|}
\hline No & Parameter & Symbol & Value \\
\hline 1 & Mass of the Helicopter & $m_{\text {quad }}$ & 1.421 \\
\hline 2 & $\begin{array}{l}\text { Equivalent Moment of Inertia about } \\
\text { Pitch Axis }\end{array}$ & $J_{p}$ & 0.0384 \\
\hline 3 & $\begin{array}{l}\text { Equivalent Moment of Inertia about } \\
\text { Yaw Axis }\end{array}$ & $J_{y}$ & 0.0432 \\
\hline 4 & $\begin{array}{l}\text { Pitch Propeller Torque-Thrust } \\
\text { Constant }\end{array}$ & $K_{p p}$ & 0.2041 \\
\hline 5 & $\begin{array}{l}\text { Pitch and Yaw Motor Voltage- } \\
\text { Torque Constant }\end{array}$ & $K_{p y}$ & 0.0068 \\
\hline
\end{tabular}




\begin{tabular}{|l|l|l|l|}
\hline 6 & $\begin{array}{l}\text { Yaw and Pitch Motor Voltage- } \\
\text { Torque Constant }\end{array}$ & $K_{y p}$ & 0.0219 \\
\hline 7 & $\begin{array}{l}\text { Yaw Propeller Torque-Thrust } \\
\text { Constant }\end{array}$ & $K_{y y}$ & 0.0720 \\
\hline 8 & $\begin{array}{l}\text { Helicopter Center of Mass from Pivot } \\
\text { along Pitch Axis }\end{array}$ & $l_{c m}$ & 0.1857 \\
\hline 9 & Pitch Viscous Damping Constant & $B_{p}$ & 0.8000 \\
\hline 10 & Yaw Viscous Damping Constant & $B_{y}$ & 0.3180 \\
\hline
\end{tabular}

$$
A=\left(\begin{array}{cccc}
0 & 0 & 1 & 0 \\
0 & 0 & 0 & 1 \\
0 & 0 & -9.2768 & 0 \\
0 & 0 & 0 & -3.4931
\end{array}\right) \quad B=\left(\begin{array}{ccc}
0 & 0 \\
0 & 0 \\
2.3667 & 0.0789 \\
0.2406 & 0.7909
\end{array}\right) \quad C=\left(\begin{array}{llll}
1 & 0 & 0 & 0 \\
0 & 1 & 0 & 0 \\
0 & 0 & 1 & 0 \\
0 & 0 & 0 & 1
\end{array}\right) \quad D=\left(\begin{array}{ll}
0 & 0 \\
0 & 0 \\
0 & 0 \\
0 & 0
\end{array}\right)
$$

\section{Adaptive Fuzzy Controller Design}

Adaptive Fuzzy Controller is designed with some adjustable parameters alongside an embedded mechanism for adjusting them. An adaptive controller has been used for improving the general overall performance of the controller [17].

The design of a controller is primarily based totally on an assumed mathematical version that resembles an actual device. The error among the real device and its mathematical illustration is calculated and if it's far fairly insignificant then the version is believed to paintings effectively.

A threshold consistent that units a boundary for the effectiveness of a controller, additionally exists. The control enter is fed into each actual device and mathematical version. Here, assumes $\mathrm{x}(\mathrm{t})$ is the output of the actual device and $\mathrm{y}(\mathrm{t})$ is the output of the mathematical version. Then the error e(t) may be calculated as follows -

$$
e(t)=x(t)-y(t)
$$

Here, $\mathrm{x}$ desired is the output we need from the device, and $\mu(\mathrm{t})$ is the output coming from the controller and going to each actual in addition to the mathematical model. The approach includes (i) Identification based learning and (ii) Fuzzy inferencing for control so the fuzzy logic control system can be adapted to the controlled system while the fuzzy logic controller simultaneously calculates and applies the control inputs to the system [18]. In Figure 2, the inner-loop achieves fuzzy logic identification (i) while the outer-loop performs fuzzy control (ii). The main objective of fuzzy logic identification here is to obtain the input/output relation of the dynamic behavior of the system. The fuzzy logic controller is a set of 'if-then rules consisting of premise fuzzy propositions and consequence fuzzy propositions. The use fuzzy hyper-cell for the premise part 
which has fixed membership functions assigned to each fuzzy cell, and will define fuzzy logic operators for the consequence part which has time-varying membership functions as adaptively inference control actions.

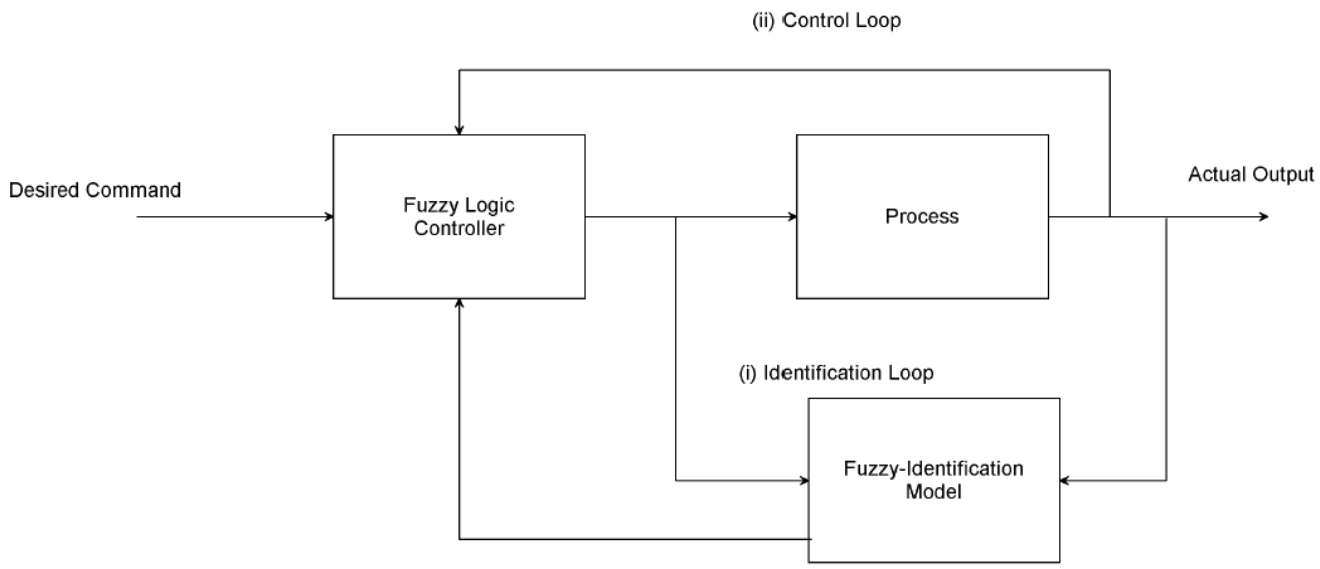

Figure 2 Adaptive Fuzzy logic control

\subsection{System Parameterization}

A fuzzy controller the layout of that's primarily based totally on the fuzzy mathematical version can have the following shape of fuzzy rules -

$$
\begin{aligned}
& \text { Rule 1-IF } \quad x_{1}\left(t_{n}\right) \in X_{11} \text { AND...AND } x_{i}\left(t_{n}\right) \in X_{1 i} \\
& \text { THEN } \mu_{1}\left(t_{n}\right)=K_{11} x_{1}\left(t_{n}\right)+K_{12} x_{2}\left(t_{n}\right)+\ldots+K_{1 i} x_{i}\left(t_{n}\right) \\
& \text { Rule 2-IF } x_{1}\left(t_{n}\right) \in X_{21} \text { AND...AND } x_{i}\left(t_{n}\right) \in X_{2 i} \\
& \text { THEN } \mu_{2}\left(t_{n}\right)=K_{21} x_{1}\left(t_{n}\right)+K_{22} x_{2}\left(t_{n}\right)+\ldots+K_{2 i} x_{i}\left(t_{n}\right) \\
& \text { Rule j-IF } x_{1}\left(t_{n}\right) \in X_{k 1} \text { AND...AND } x_{i}\left(t_{n}\right) \in X_{k i} \\
& \text { THEN } \mu_{j}\left(t_{n}\right)=K_{j 1} x_{1}\left(t_{n}\right)+K_{j 2} x_{2}\left(t_{n}\right)+\ldots+K_{j i} x_{i}\left(t_{n}\right)
\end{aligned}
$$

The on the top set of parameters characterizes the controller.

\subsection{Controller Adjustment}

The controller parameters are adjusted to enhance the overall performance of the controller. The system of calculating the adjustment to the parameters is the adjusting mechanism. Mathematically, permit $\theta^{(n)}$ be hard and fast of parameters to be adjusted at time $\mathrm{t}=\mathrm{tn}$. The adjustment may be the recalculation of the parameters,

$$
\theta^{(n)}=\Theta\left(D_{0}, D_{1}, \ldots, D_{n}\right)
$$

Here $\mathrm{Dn}$ is the data collected at time $\mathrm{t}=\mathrm{tn}$ 
Now, this formulation is reformulated by the update of the parameter set supported its previous worth as

$$
\theta^{(n)}=\phi\left(\theta^{n-1}, D_{n}\right)
$$

\subsection{Adaptive Fuzzy Controller Parameter Selection}

The following parameters need to be thought of for choosing AN reconciling fuzzy controller -

- Can the machine be approximated totally with the aid of using a fuzzy version?

- If a machine may be approximated totally with the aid of using a fuzzy version, are the parameters of this fuzzy version effortlessly to be had or need to be decided online?

- If a machine can't be approximated totally with the aid of using a fuzzy version, can or not it's approximated piecewise with the aid of using a fixed fuzzy version?

- If a machine may be approximated with the aid of using a set of fuzzy fashions, are those fashions having the identical layout with extraordinary parameters or are they having extraordinary formats?

- If a machine may be approximated with the aid of using a set of fuzzy fashions having the identical layout, every with an extraordinary set of parameters, are those parameter units effortlessly to be had or need to they be decided online?

\subsection{Fuzzy Logic Controller}

Fuzzy logic is applied with nice success in the varied control application. most of the buyer products have fuzzy control. a control system is a meeting of physical components designed to change another physical system so this technique exhibits sure desired characteristics. whereas applying traditional control, one has to fathom the model and therefore the objective performs developed in precise terms. This makes it troublesome to use in several cases. By applying Fuzzy logic for control, we tend to can utilize the human expertise and talent for designing a controller. The fuzzy control rules, essentially the IF-THEN rules, can be best used in coming up with a controller. For the fuzzy logic mechanism, the signal variables are error (e) and derivative of error (de/dt), and the output. Gaussian membership functions are utilized for input variables and the output. An error has 9 membership functions as shown in Figure 3, a derivative of error has 9 membership functions as shown in Figure 4, and output has nine membership functions as shown in Figure 5 below. 


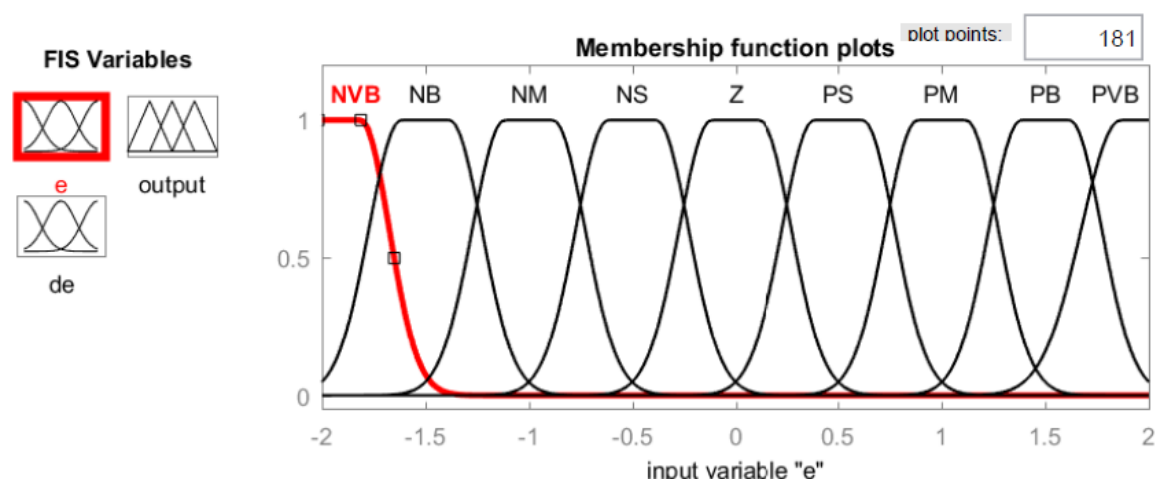

Figure 3 Error membership function

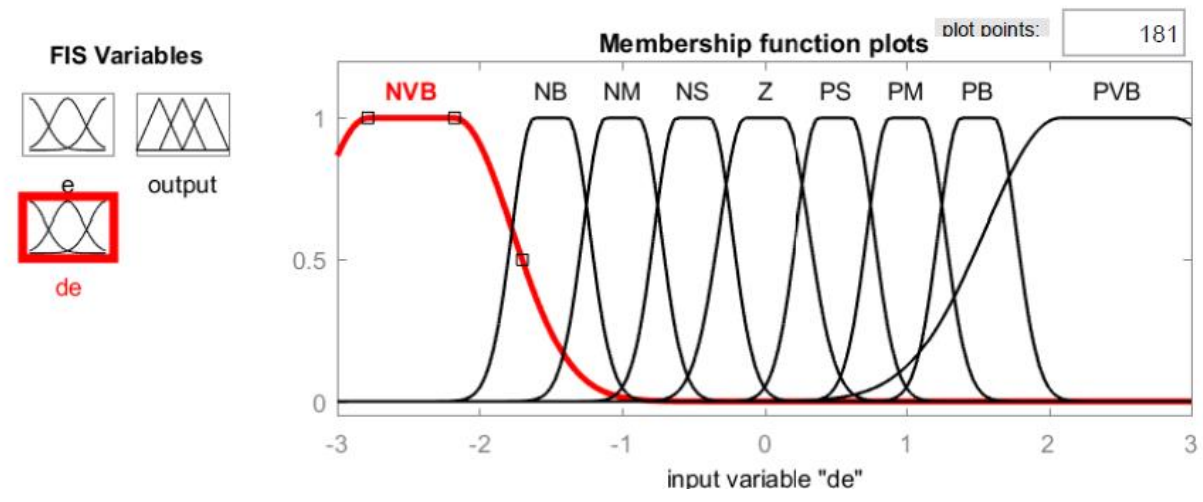

Figure 4 Change of error membership function
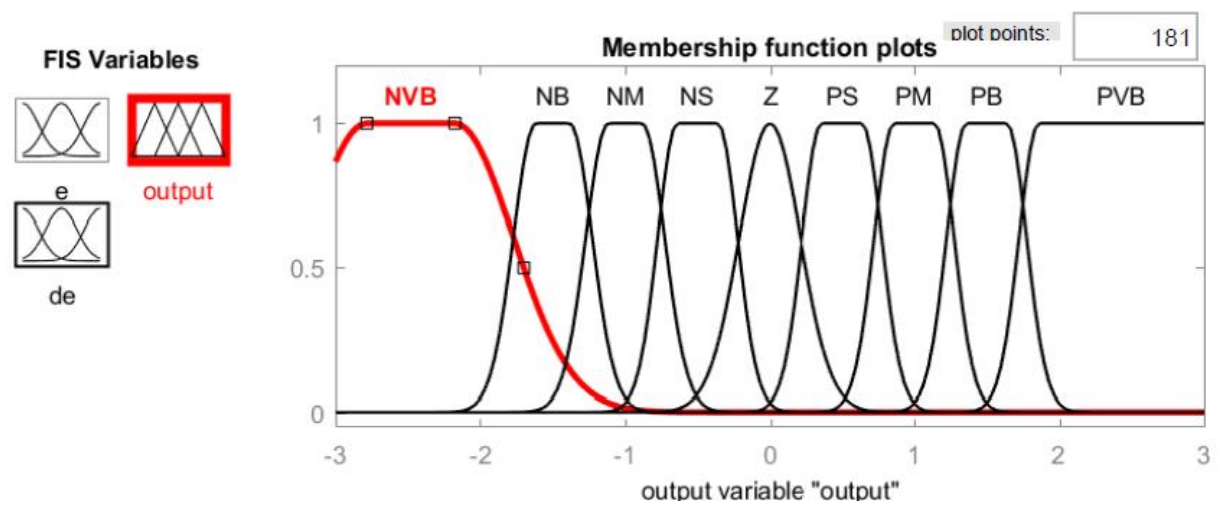

Figure 4 Output membership function

\subsubsection{Fuzzy Rule Base}

The fuzzy input variable e has nine membership functions and fuzzy input variable $\Delta \mathrm{e}$ has nine membership functions, and the output variable has nine membership functions. There are 81 rules generated as shown in Table 2 .

Table 2 Fuzzy rule base

$$
\Delta e
$$




\begin{tabular}{|l|l|l|l|l|l|l|l|l|l|l|}
\hline \multicolumn{2}{|c|}{$\mathrm{u}$} & NVB & NB & NM & NS & Z & PS & PM & PB & PVB \\
\hline \multirow{6}{*}{$e$} & NVB & PVB & PVB & PVB & PB & PM & PM & PS & Z & Z \\
\cline { 2 - 11 } & NB & PVB & PVB & PB & PM & PS & PS & PS & Z & Z \\
\cline { 2 - 11 } & NM & PVB & PB & PM & PS & PS & Z & Z & Z & NS \\
\cline { 2 - 11 } & NS & PB & PM & PM & PS & PS & Z & Z & NS & NS \\
\cline { 2 - 11 } & Z & PM & PM & PS & Z & Z & Z & NS & NS & NM \\
\cline { 2 - 11 } & PS & PM & PS & PS & Z & NS & NS & NM & NM & NB \\
\cline { 2 - 11 } & PM & PS & PS & Z & NS & NS & NM & NB & NB & NB \\
\cline { 2 - 11 } & PB & PS & Z & Z & NS & NM & NM & NB & NVB & NVB \\
\cline { 2 - 11 } & PVB & Z & $Z$ & NS & NM & NM & NB & NB & NVB & NVB \\
\hline
\end{tabular}

\section{Simulation Results}

To validate the approach used, we carried out simulations for the adaptive fuzzy control of a 2DOF Quadrotor moving along a specified trajectory, whose model parameters are estimated from manufactured quadrotors. The quadrotors are commanded to track a square wave pitch and yaw angle trajectory through a function of time. The control performance obtained by the proposed control system is compared with Fuzzy and PID controllers to validate the superior performance of the studied controller. A comparison of the adaptive fuzzy controller was carried out with Fuzzy and PID controllers. The results of this comparison confirmed the improvement in the performance of the proposed controller. The PID-based control was used to control the quadrotor for a control purpose. The simulation results are illustrated in Figure 10. 


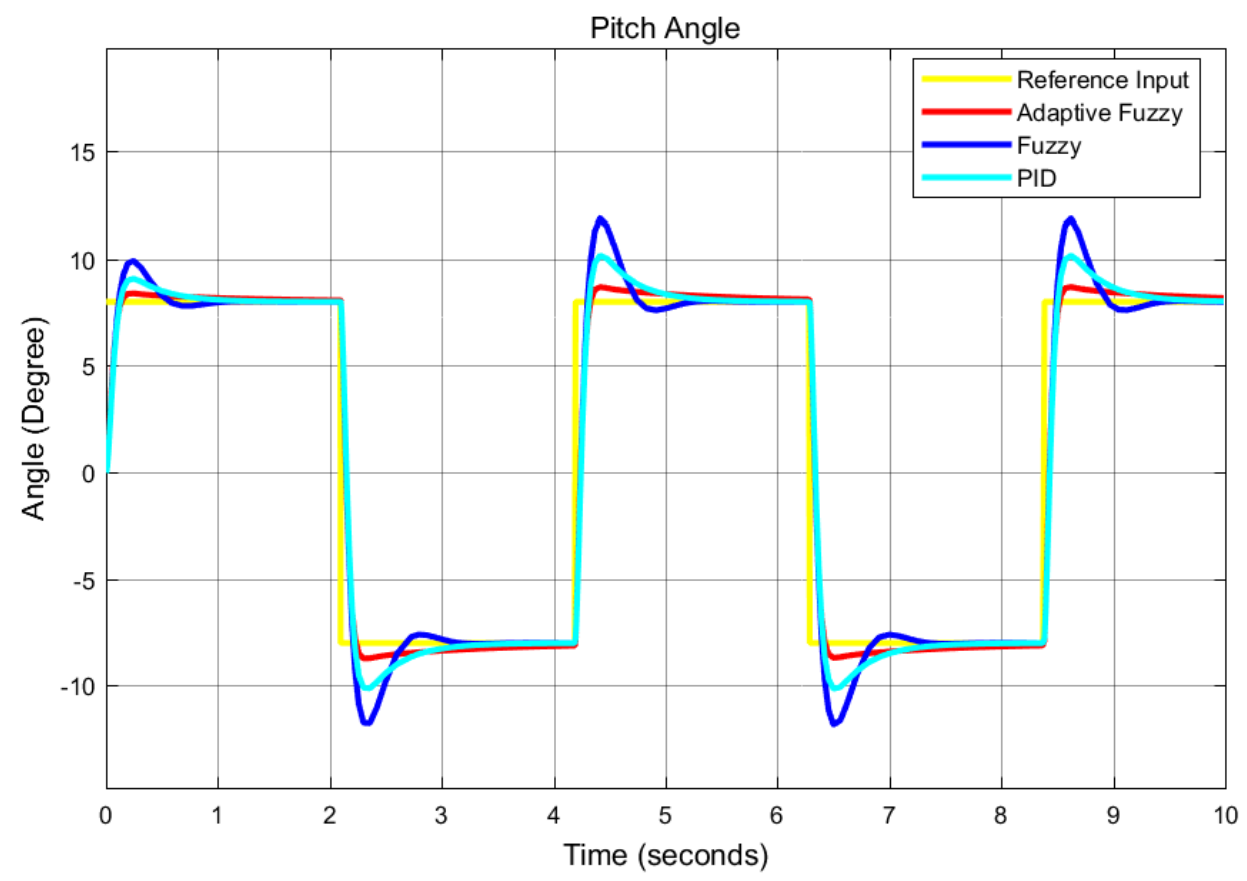

Figure 10 Pitch angle response to reference pitch angle input

The reference pitch angle position trajectory is compared with the measured one for the adaptive fuzzy, Fuzzy, and PID as shown in Figure 10. As we can see in Figure 10, the PID controller can reproduce the tracking trajectory with an error of $12.5 \%$. The control by Fuzzy controller has proved its worth. The effect of the pitch angle trajectory over the measured yaw angle trajectory is shown in Figure 11 below.

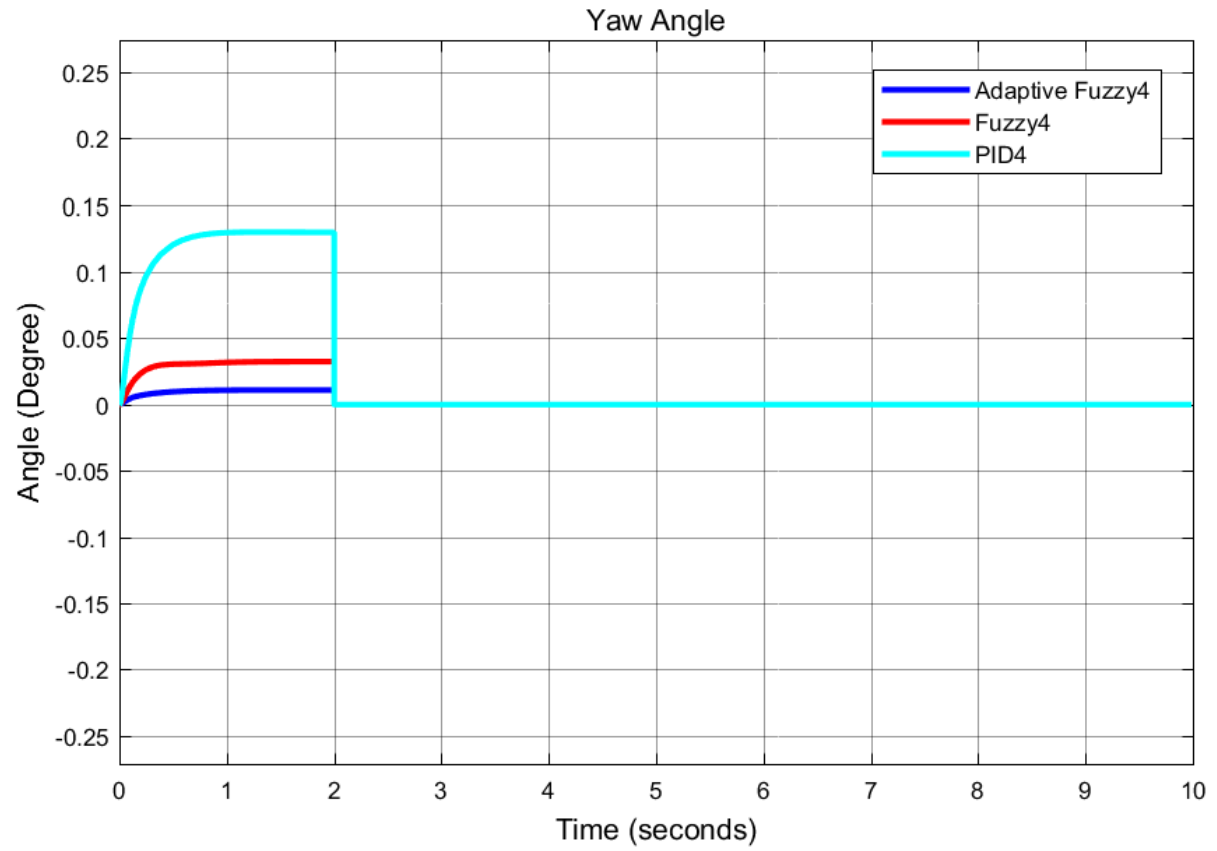

Figure 11 Yaw angle response to reference pitch angle input

The simulation result shows that there is a small angle trajectory caused by the effect of the desired reference pitch angle and the result of this comparison confirmed the improvement in the performance of the proposed controller. 
Based on the simulation results obtained in Figure 12, the results indicate that the reference yaw angle position trajectory of the quadrotor track is compared with the measured one for the adaptive fuzzy, Fuzzy, and PID controllers. The adaptive fuzzy control actual trajectory compared with the yaw angle reference has improved the performance of the quadrotor better than the Fuzzy and PID controllers.

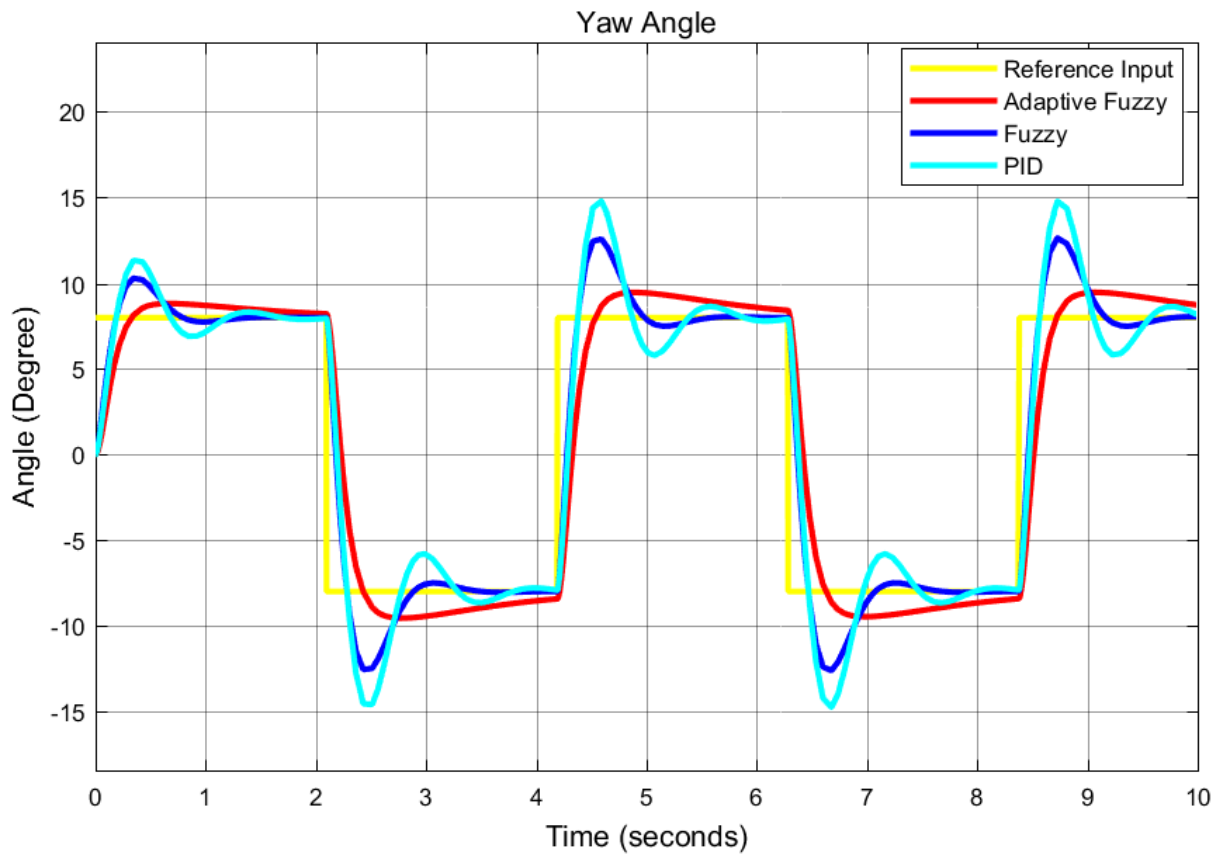

Figure 12 Yaw angle response to reference yaw angle input

The effect of the yaw angle trajectory over the measured pitch angle trajectory is shown in Figure 13 below. The simulation result shows that there is a small angle trajectory caused by the effect of the desired reference yaw angle and the result of this comparison confirmed the improvement in the performance of the proposed controller.

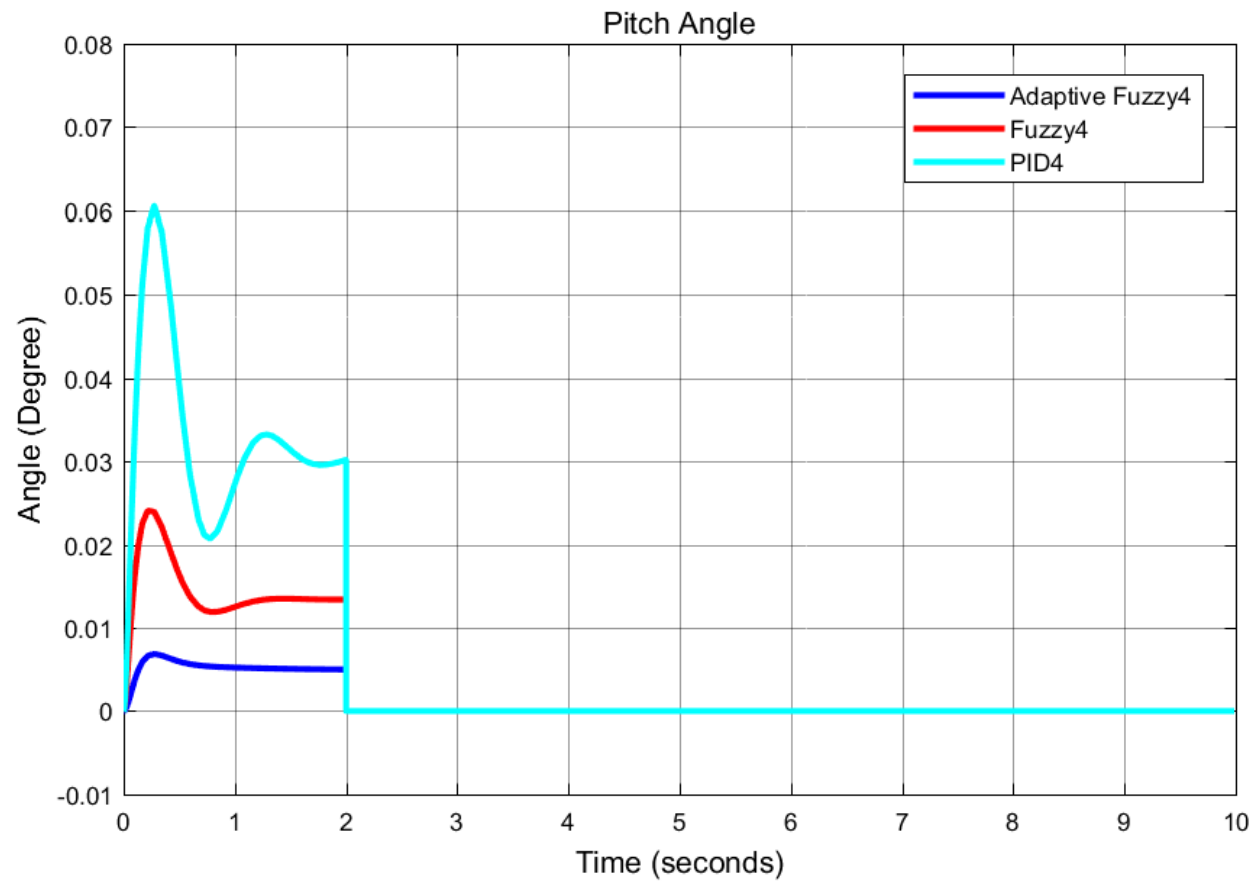


Figure 13 Pitch angle response to reference yaw angle input

\section{Conclusion}

This research was dedicated to solving the limitations of classical control laws using modern control theory. The use of control methods based on intelligence techniques has become a necessity. These techniques include neural networks, fuzzy logic, and evolutionary algorithms, among many others. The paper presents an adaptive control theory applied to fit a Fuzzy logic system membership function. The case study was implemented using simulations, whose main purpose is to control a 2 DOF quadrotor trajectory tracking task using MATLAB Simulink Toolbox. To validate this intelligent approach, a Gaussian membership function was used to optimize the Fuzzy parameters via the adaptive control theory. The results obtained were compared with a Fuzzy, and PID, to prove the high performance of the Adaptive Fuzzy logic controller.

\section{Reference}

[1].Zexin WANG, Jiang ZHAO, Zhihao CAI, Yingxun WANG, Ningjun LIU. (2021) Onboard actuator model-based Incremental Nonlinear Dynamic Inversion for quadrotor attitude control: Method and application. Chinese Journal of Aeronautics 32.

[2].Vazquez-Nicolas, J.M., Zamora, E., González-Hernández, I. et al. PD+SMC Quadrotor Control for Altitude and Crack Recognition Using Deep Learning. Int. J. Control Autom. Syst. 18, 834-844 (2020). https://doi.org/10.1007/s12555-018-0852-9

[3].W. Craig, D. Yeo, and D. A. Paley, "Geometric Attitude and Position Control of a Quadrotor in Wind," J. Guid. Control. Dyn., vol. 43, no. 5, pp. 870-883, 2020, DOI: 10.2514/1. G004710.

[4].Hassani Hassani, Anass Mansouri, Ali Ahaitouf. (2020) A new robust adaptive sliding mode controller for the quadrotor UAV flight. 2020 IEEE 2nd International Conference on Electronics, Control, Optimization and Computer Science (ICECOCS), pages 1-6.

[5]. Bowen Xu, Xinjiang Lu. (2020) An Online Adaptive Control Strategy for Trajectory Tracking of Quadrotors Based on Fuzzy Approximation and Robust Sliding Mode Algorithm. IEEE Access 8, pages 215327-215342.

[6].Zhi Li, Xin Ma \& Yibin Li (2020) Robust trajectory tracking control for a quadrotor subject to disturbances and model uncertainties, International Journal of Systems Science, 51:5, 839-851, DOI: 10.1080/00207721.2020.1746430

[7]. Chu Z, Zhou S, Zhu M, Li H. Finite-time trajectory control for a quadrotor aircraft using disturbance observer. International Journal of Advanced Robotic Systems. March 2020. doi: $10.1177 / 1729881420903847$

[8].Ha SW, Park BS. Disturbance Observer-Based Control for Trajectory Tracking of a Quadrotor. Electronics. 2020; 9(10):1624. https://doi.org/10.3390/electronics9101624

[9].Y. I. Jennie, A. Fathurrahman, O. Arifianto, and R. A. Sasongko, "Mathematical modeling and simulation of a quadrotor unmanned aerial vehicle with automatic altitude and speed control", AIP Conference Proceedings 2226, 020011 (2020) https://doi.org/10.1063/5.0002805

[10]. Mustefa Jibril Taha, F. B. Kibret, V. Ramayya, and B. A. Zeru, "Design and evaluation of solar parabolic trough collector system integrated with conventional oil 
boiler," Arch. Electr. Eng., vol. 70, no. No 3, pp. 657-673, 2021, doi:

10.24425/aee.2021.137580.

[11]. Kim, Young-Min, et al. "Gyro Effect Control of the Quadrotor UAV Using Feedback Linearization." Journal of Korea Robotics Society, vol. 15, no. 3, The Korea Robotics Society, July 2020, pp. 248-255. Crossref, doi:10.7746/jkros.2020.15.3.248.

[12]. M. A. Lotufo, L. Colangelo and C. Novara, "Control Design for UAV Quadrotors via Embedded Model Control," in IEEE Transactions on Control Systems Technology, vol. 28, no. 5, pp. 1741-1756, Sept. 2020, DOI: 10.1109/TCST.2019.2918750.

[13]. Hyukwoo Lee, Kyunghyun Lee, and Kwanho You. 2019. Quadrotor Stabilization and Tracking using Sliding Mode Observer and Control. In Proceedings of the 2019 7th International Conference on Computer and Communications Management (ICCCM 2019). Association for Computing Machinery, New York, NY, USA, 28-31.

DOI:https://doi.org/10.1145/3348445.3348476

[14]. J. Kim, S. A. Gadsden, and S. A. Wilkerson, "A Comprehensive Survey of Control Strategies for Autonomous Quadrotors," in Canadian Journal of Electrical and Computer Engineering, vol. 43, no. 1, pp. 3-16, winter 2020, DOI: 10.1109/CJECE.2019.2920938.

[15]. He Z H, Gao W L, He X K, Wang M J, Liu Y L, Song Y, et al. Fuzzy intelligent control method for improving flight attitude stability of plant protection quadrotor UAV. Int J Agric \& Biol Eng, 2019; 12(6): 110-115.

[16]. G. Yu, D. Cabecinhas, R. Cunha and C. Silvestre, "Nonlinear Backstepping Control of a Quadrotor-Slung Load System," in IEEE/ASME Transactions on Mechatronics, vol. 24, no. 5, pp. 2304-2315, Oct. 2019, DOI: 10.1109/TMECH.2019.2930211.

[17]. Malik, W., Hussain, S. Developing of the smart quadcopter with improved flight dynamics and stability. Journal of Electrical Systems and Inf Technol 6, 6 (2019). https://doi.org/10.1186/s43067-019-0005-0

[18]. Yang, L., Chen, Y., Liu, Z. et al. Adaptive Fuzzy Control for Teleoperation System with Uncertain Kinematics and Dynamics. Int. J. Control Autom. Syst. 17, 11581166 (2019). https://doi.org/10.1007/s12555-017-0631-z

[19]. C. Wu, J. Liu, X. Jing, H. Li, and L. Wu, "Adaptive Fuzzy Control for Nonlinear Networked Control Systems," in IEEE Transactions on Systems, Man, and Cybernetics: Systems, vol. 47, no. 8, pp. 2420-2430, Aug. 2017, DOI: 10.1109/TSMC.2017.2678760. 